\title{
Bioanalysis
}

\section{Imaging mass spectrometry in drug discovery and development}

\author{
"The strength of MSI lies in its ability to preserve the spatial location of \\ molecular entities in tissues while maintaining the molecular specificity of mass \\ spectrometry."
}

Keywords: 3D imaging $\bullet$ drug development $\bullet$ drug discovery $\bullet$ drug distribution $\bullet$ metabolite distribution $\bullet$ MS imaging $\bullet \mathrm{MSI}$

This themed issue was compiled to give pharmaceutical researchers, be it analytical, biological or chemical, a brief but current review on mass spectrometry imaging (MSI) technology, methods and applications as used in pharmaceutical research and development, including current challenges and future developments. The strength of MSI lies in its ability to preserve the spatial location of molecular entities in tissues while maintaining the molecular specificity of mass spectrometry. As such, this technology has a unique place in pharmaceutical research and development to provide spatial distribution of unlabeled compounds and their metabolites as well as potential biomarkers of efficacy and toxicity. This unique ability lends to its utility across all aspects of research and development, from target occupancy and drug metabolism and pharmacokinetics, to pharmacodynamics and toxicity. These distinctive aspects and applications of MSI for pharmaceutical research and development have been reviewed in the following articles.

The articles within this issue are split into three categories. The first section offers a review of the basic concepts of MSI. The article by Andren et al. provides a brief introduction to imaging, including the principals, instrumentation and experimental procedures [1]. It also introduces the reader to the subject of drug quantitation and the various pharmaceutical research and development focus areas where imaging has played an important role (e.g., drug metabolism and safety toxicity). Garrett et al. then provide an overview of ionization sources and mass analyzers that are used for tissue-based MSI [2]. In the second section, example applications are reviewed to illustrate the diverse role of MSI in pharmaceutical research and development. The article by Takai et al. focuses on case studies of imaging drug and metabolite distribution [3], whereas Drake et al. [4] present studies in which MSI was employed to discover biomarkers of drug efficacy. Lastly, emerging developments and their impact are discussed. Pirman [5] provides an overview of different analytical approaches for quantitative MSI and an introduction to 3D imaging and depth profiling is discussed by Fletcher [6]. Lastly, Goodwin et al. discuss current and forward facing opportunities as well as challenges for MSI and its role in pharmaceutical research and development, including regulatory implications [7].

There have been exciting recent developments in MSI, leading to increased capabilities; however, there are still many unmet possibilities for MSI to impact pharmaceutical research and development. With additional improvements in spatial resolution, drug localization at the cellular level may be possible and this is currently an essential information gap in pharmaceutical research. Furthermore, additional quantitative developments will facilitate quantitative drug imaging for both whole body and tissue substructure distribution studies using nonradiolabeled compound, making these studies feasible earlier in the discovery and development process. Methods for detecting larger therapeutics, such as antibodies, proteins and oligonucleotides, will expand the applicability of MSI to biotherapeutics and molecules that are not well suited

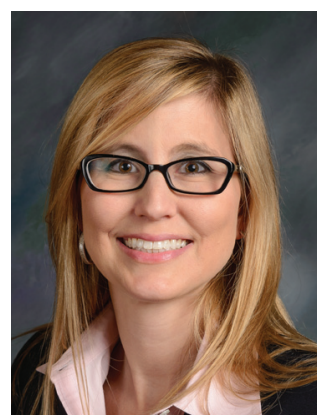

Stacey R Oppenheimer Author for correspondence: Center of Chemistry Innovation and Excellence, Pfizer, Inc., Groton, CT, USA staceyopp@gmail.com

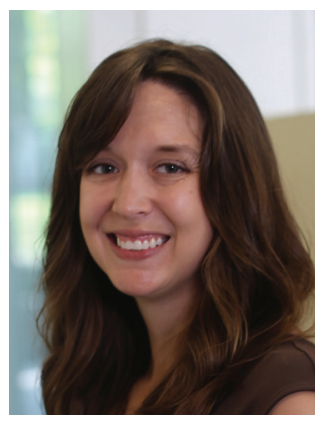

Angela Y Wehr

Drug Metabolism and Pharmacokinetics, Biogen, Cambridge, MA, USA 
to radiolabeling. As our ability to process large quantities of data improve, 3D imaging and high-resolution imaging of larger specimens will allow us to explore samples in greater detail and these capabilities are currently being developed. As these improvements are achieved, MSI will likely be more commonly accepted and utilized by the pharmaceutical industry. The routine application of these methods will enable distribution studies to be conducted faster and at a lower cost, ultimately driving drug discovery and development.

\section{References}

1 Lodén H, Shariatgorji M, Nilsson A, Andrén PE. An introduction to mass spectrometry imaging in drug discovery and development. Bioanalysis 7(20), 2621-2627 (2015).

2 Tsai YH, Menger RF, Drexler DM, Yost RA, Garrett TJ. Bioanalysis 7(20), 2629-2637 (2015).

3 Takai N, Tanaka Y. Imaging of drug and metabolite distribution by mass spectrometry: case studies. Bioanalysis 7(20), 2639-2648 (2015).

\section{Financial \& competing interests disclosure}

The authors have no relevant affiliations or financial involvement with any organization or entity with a financial interest in or financial conflict with the subject matter or materials discussed in the manuscript. This includes employment, consultancies, honoraria, stock ownership or options, expert testimony, grants or patents received or pending, or royalties.

No writing assistance was utilized in the production of this manuscript.

4 Jones EE, Gao P, Smith CD, Norris JS, Drake RR. Tissue biomarkers of drug efficacy: case studies using a MALDI-MSI workflow. Bioanalysis 7(20), 2611-2619 (2015).

5 Pirman D. Quantitative profiling tissue drug distribution by mass spectrometric imaging (MSI). Bioanalysis $7(20)$, 2649-2656 (2015).

6 Fletcher J. 3D imaging of biological specimen using mass spectrometry. Bioanalysis 7(20), 2657-2666 (2015).

7 Goodwin RJ. Future directions of imaging mass spectrometry in pharmaceutical R\&D. Bioanalysis 7(20), 2667-2673 (2015). 